\title{
EFICIENCIA TÉCNICA RELATIVA EN LA PRESTACIÓN DEL SERVICIO DE TRANSPORTE PÚBLICO EN COSTA RICA. RUTAS AUTOBUSERAS INTERREGIONALES, 2009
}

\author{
David Navarro-Rodríguez ${ }^{1}$ \\ Jorge Hidalgo-Rojas ${ }^{2}$
}

\begin{abstract}
RESUMEN
Este artículo presenta una aplicación de modelos de frontera para la evaluación de la eficiencia técnica relativa del sector regulado del transporte público en Costa Rica. Se elaboró un modelo de análisis envolvente de datos (DEA) para calcular la eficiencia técnica relativa en la prestación de servicio de una muestra de rutas interregionales a nivel nacional, a partir de una encuesta realizada sobre los operadores, por parte de la Autoridad Reguladora de Servicios Públicos (ARESEP) en el año 2009. Como variables de producto a evaluar se utilizaron los promedios mensuales de pasajeros por kilómetro recorrido $y$ de pasajeros por bus. Como variables de insumo se consideraron los gastos devengados en diferentes aspectos clave para la prestación del servicio. A partir del modelo, se evaluó el desempeño de las 29 rutas y se determinaron los mecanismos de mejora para aquellas clasificadas como ineficientes.
\end{abstract}

PALABRAS CLAVE: GESTIÓN DE RECURSOS, SERVICIOS DE UTILIDAD PÚBLICA, ANÁLISIS INSUMO-PRODUCTO, PROGRAMACIÓN LINEAL, TRANSPORTE PÚBLICO.

\section{ABSTRACT}

This article represents an application of frontier models for the evaluation of the relative technical efficiency in the regulated public transport sector in Costa Rica. Using data from a survey made by Autoridad Reguladora de Servicios Públicos (ARESEP) in 2009 a data envelopment analysis (DEA) model was computed to obtain the relative efficiency for the public service provided in interregional public transit routes around the country. As output variables, the number of passenger for kilometer of travel and monthly average of passenger per bus were used. As input variables, we used the spending of many key aspects to provide the service. Based on the model, the 29 interregional routes were classified and ranked by their respective efficiency. In addition, for those classified as inefficient, some enhancement factors were provided.

KEYWORDS: RESOURCE MANAGEMENT, PUBLIC UTILITIES, INPUT-OUTPUT ANALYSIS, LINEAR PROGRAMMING, PUBLIC TRANSPORT.

1 Universidad de Costa Rica, Instituto de Investigaciones en Ciencias Económicas; Código postal 115012060; San José, Costa Rica; david.navarrorodriguez@ucr.ac.cr

2 Universidad de Costa Rica, Instituto de Investigaciones en Ciencias Económicas; Código postal 115012060; San José, Costa Rica; j.hidalgo90@hotmail.com 


\section{INTRODUCCIÓN}

En los últimos años, el transporte público se ha ligado estrechamente con aspectos relacionados a largo plazo con el desarrollo de un país, como la competitividad, el crecimiento económico, la dinámica de la sociedad en los centros poblacionales y la calidad de vida de sus habitantes. En ese sentido, el procurar una estructura apropiada de la prestación del servicio, de acuerdo con las necesidades sociales y económicas presentes, y que garantice la calidad al usuario, son puntos elementales dentro de las metas de políticas públicas de un país ${ }^{3}$ que deben ser parte de los lineamientos de regulación que rigen tal servicio. En ese marco es que la Administración Pública, basada en el bien común, define una serie de medidas para el servicio regulado, las cuales incluyen la fijación de precios, así como un nivel mínimo de garantías del servicio prestado, como la determinación de la frecuencia de salida de las unidades de la terminal, que podría justificarse más a un nivel de interés social que económico.

De Borger, Kerstens y Costa (2002) consideran que, en este marco, la eficiencia se ha convertido en uno de los elementos relevantes para la toma de decisiones. Numerosos mecanismos se han utilizado para medir la eficiencia en procesos productivos, en donde los estudios de frontera han ido tomando interés, particularmente por la capacidad de facilitar las comparaciones entre la prestación del servicio por un agente $y$ otro (benchmarking) $y$ las facilidades que provee al caracterizar la tecnología utilizada por estos en el proceso $0^{4}$.

En este artículo, se presenta una aplicación de los modelos de frontera, particularmente el modelo de análisis envolvente de datos (DEA, por sus siglas en inglés), al sector de transporte público en Costa Rica. Este es un modelo que no se ha utilizado previamente a nivel nacional para evaluar el desempeño relativo de los operadores que prestan este servicio, pese a que en las últimas décadas se ha dado un aumento importante de estudios en otros países, utilizando esta metodología u otros modelos de frontera para el análisis del transporte público ${ }^{5}$.

El modelo se aplica sobre diferentes rutas de tipo interregional, a nivel nacional, las cuales se definen de acuerdo con LCR Logística (2002) como aquellas que comunican dos o más regiones con áreas metropolitanas diferentes ${ }^{6}$. Para ello, se utilizaron datos obtenidos por una encuesta realizada en el 2009 por ARESEP ${ }^{7}$ a las empresas operadoras del servicio concesionado o con permisos. Bajo la hipótesis inicial de que las rutas evaluadas presentan distintos niveles de eficiencia en el uso de recursos para la prestación del servicio, a partir del modelo, se exponen los niveles de eficiencia de cada una de las rutas interregionales, clasificándolas entre eficientes e ineficientes, $y$ definiendo su posición dentro del conjunto de rutas evaluadas (ranking); además, se proponen factores de mejoras, basados en las prácticas más sobresalientes observadas dentro de la muestra, para aquellas clasificadas como ineficientes.

Todo lo anterior se deriva de un subconjunto de los resultados obtenidos por los autores como parte de su tesis de licenciatura en Economía (Hidalgo \& Navarro, 2016), y se realiza con el fin de mostrar la aplicabilidad de modelos de esta naturaleza a sectores productivos regulados por el Estado, particularmente al sector del transporte público en modalidad de autobús.

El desarrollo de este artículo versa de la siguiente manera: en el siguiente apartado se discute la metodología empleada, la definición del modelo, la selección de variables y rutas por evaluar. Posteriormente, se analizan los resultados del sector, se clasifican las rutas según el modelo DEA y se aplican, para una de las rutas, los ajustes de mejora correspondientes. Además, se reflexiona sobre la aplicación de modelos de este tipo en un esquema de regulación de transporte público.

3 Ver como referencia para Costa Rica, el Plan Nacional de Desarrollo 2015-2018 y el Plan Nacional de Transporte 2011-2035.

4 Para más información sobre la evolución de las metodologías para medir productividad y eficiencia, Daraio y Simar (2007) reflexionan brevemente sobre la historia del pensamiento y las metodologías el análisis de eficiencia relativa.

$5 \quad$ Para fines referenciales, Karlaftis et al. (2013) compila un conjunto amplio de investigaciones académicas realizadas en las que se ha aplicado alguna variación se modelo DEA en el sector de transporte público, entre los años 1990 y 2013 (al primero de abril).

6 La escogencia de este conjunto de rutas es por un interés particular basado en un conocimiento a priori sobre la homogeneidad de las condiciones a las que están sujetos los operarios de las rutas respecto a la prestación del servicio.

$7 \quad$ La Autoridad Reguladora de Servicios Públicos es la entidad encargada de regular los servicios públicos a nivel nacional, que incluyen el suministro de energía eléctrica (etapas de generación, transmisión, distribución y comercialización), telecomunicaciones , suministro del servicio de acueducto y alcantarillado, suministro de combustibles derivados de hidrocarburos, transporte de carga por ferrocarril, recolección y tratamiento de desechos sólidos e industriales, servicios marítimos y aéreos en puertos nacionales y cualquier medio de transporte público remunerado de personas, exceptuando el aéreo (artículo 5 de la Ley No. 7593, Ley de la Autoridad Reguladora de Servicios Públicos, del 9 de agosto de 1996). 


\section{METODOLOGÍA}

\section{Escogencia del modelo}

El concepto en el que se basa este análisis es el de eficiencia técnica, la cual se compone de dos elementos: eficiencia técnica pura y eficiencia de escala. La primera evalúa la implementación de un plan de producción al convertir insumos en productos; mientras que la segunda evalúa la divergencia sobre el tamaño de escala de producción, con lo cual se posibilita obtener datos sobre las principales fuentes de ineficiencia que pudiesen presentarse (Garcia, 2009).

La investigación, como ya se mencionó, hace uso del método denominado DEA, planteado por primera vez por Charles, Cooper y Rhodes (1978), el cual se aplica al estudio de la frontera de producción y consiste en el desarrollo de un esquema de optimización no paramétrica que permite identificar el grado de eficiencia técnica relativa entre un conjunto de unidades decisoras en el sector productivo (Decision Market Unit - DMU), las cuales son de naturaleza homogénea.

Tal método supone rendimientos a escala constantes (RCE), lo que implicaría que la prestación del servicio se vea condicionada en términos de eficiencia -de igual forma- por factores tales como: el tipo de relieve, el estado de las carreteras y nivel de congestionamiento vehicular, así como la altura sobre el nivel de mar, aunque sean elementos que en realidad afectan de manera muy diferente a las rutas y que corresponden a variables que no se consideran de manera explícita en el presente artículo. Además, el tamaño de las empresas puede cambiar pues, aunque todas las rutas bajo estudio realizan recorridos interregionales, las distancias recorridas varían, al igual que la demanda a la que responden y su estacionalidad (determinante en la definición del tamaño de la flotilla, los planteles $y$ las frecuencias de salida de las unidades de las terminales, entre otros).

Con el fin de considerar los factores anteriores, se aplica además una variación del modelo denominada DEA-BCC (en honor a Banker, Charles y Cooper), que supone rendimientos variables a escala (RVE); además, permite evaluar si las DMU están operando a una escala óptima ${ }^{8}$. Así, a través de la razón entre los niveles de eficiencia bajo los modelos DEA-CCR y DEA-BCC, se obtiene el índice de eficiencia de escala para cada DMU. Dado lo anterior, se comparan los resultados de ambos modelos y se determina el tipo de rendimiento a escala que finalmente debe ser asumido para brindar las conclusiones del presente artículo.

Cabe señalar, de forma adicional, que el modelo debe definirse en términos de su orientación, es decir, respecto a qué parte del proceso productivo se considera que está bajo el control del agente tomador de decisiones, en concordancia con el enfoque de eficiencia que se desea medir. Para el presente caso, se definió que, dado que la Administración Pública define ciertos parámetros mínimos a cumplir por parte de los operadores, estos tienen predefinidos los objetivos (productos) que deben alcanzarse, por lo cual lo que finalmente tienen bajo su control son los insumos. Por tanto, el nivel de producto se toma como dado, la especificación seleccionada debe orientarse a los insumos y los ajustes para la mejora de la eficiencia relativa deben establecerse a partir de la cesta de insumos utilizada.

A nivel formal, el modelo DEA-BCC se especifica de la siguiente forma. Sea $I$ el número de DMU de una industria o sector productivo, $N$ el número de variables de insumos y $M$ el número de productos presentes en dichas DMU, donde $Y$ es una matriz $M x 1$ que representa los datos de los productos de todas las DMU y $X$ es una matriz de tamaño $N x 1$ con la información equivalente en insumos; además $y_{i}$ y $x_{i}$ son los vectores de productos $y$ de insumos de la $i$-ésima DMU, respectivamente. El supuesto de RVE es expresado por la condición de convexidad $I 1^{\prime} \times \lambda=1$, donde $I 1$ es una matriz de tamaño $I x 1$, compuesta por valores de 1 . Considerando esto, se resuelve el siguiente programa lineal para cada una de las DMU (Coelli, Passada, O’Donnell, \& Battese, 2005, p. 172):

$$
\begin{gathered}
\forall i=1, \ldots, I \\
\min _{\lambda, \theta_{i}} \theta_{i} \\
s . a . \\
Y \lambda-y_{i} \geq 0 \\
\theta x_{i}-X \lambda \geq 0 \\
I 1^{\prime} \times \lambda=1 \\
\lambda \geq 0
\end{gathered}
$$

8 Como consecuencia de esto, solo se evalúa la eficiencia técnica pura. 
Donde $\lambda$ es un vector de tamaño Ix1 y su $j$-ésimo elemento representa la intensidad de la $j$-ésima DMU.

El escalar $\theta_{i} \in[0,1]$ (coeficiente de eficiencia) corresponde a la medida de eficiencia técnica relativa, siendo simultáneamente la función objetivo y una variable por optimizar. Si $\theta_{i}^{*}=1$, como resultado del proceso de optimización, entonces la $i$-ésima DMU será, respecto a las demás, eficiente en el sentido de Farrell (1957). Por el contrario, si $\theta_{i}<1$, la DMU será considerada ineficiente, debido a que, de acuerdo con las prácticas observadas en la muestra, esta puede lograr una mejor combinación de insumo/producto. De esta forma, el modelo DEA provee información para identificar las DMU eficientes y las ineficientes, y poder clasificarlas (ranking) según coeficiente ${ }^{9}$.

Aunado a lo anterior, para las DMU ineficientes, a partir de los $\lambda_{j}>0$, se puede obtener al menos una combinación lineal de las asignaciones de insumos de las DMU eficientes que se desempeñan mejor que la evaluada (este conjunto de referencia se conoce como pares de comparación). De esta forma, el modelo provee insumos prácticos (basados en las otras DMU evaluadas) para identificar, además, el uso de insumos en los que la DMU está asignando de forma ineficiente, como también las mejoras necesarias para aumentar su rendimiento y ser eficiente bajo los criterios mencionados.

Para lograr esto, primero se deberán realizar movimientos de las variables de insumo hacia la isocuanta (con el fin de alcanzar aquella que garantice una utilización óptima de la combinación de factores productivos), lo cual se logra con una reducción de todos los insumos en una proporción de (1- $\left.\theta_{i}^{*}\right)$ manteniendo constante la cuantía de los productos. Este primer ajuste se conoce como movimiento radial (de los insumos, dada la orientación asumida). En segundo lugar, a partir de las variables de holgura (los pesos que permiten identificar la cantidad que puede reducirse un insumo específico o aumentarse un producto en particular), se pueden realizar variaciones sobre la misma isocuanta, lo cual se conoce como movimiento de holgura. Así, la mejora potencial es la dada por el cambio del movimiento radial más el de holgura para los factores productivos.

Por otro lado, en lo correspondiente a la revisión bibliográfica realizada, se identifica que para el caso de aplicación de la metodología al tema del transporte público en la modalidad de autobús, De Borger et al. (2002) identificaron un predominio de los estudios de casos europeos y estadounidenses; excepción de lo de anterior son los trabajos de Sampaio, Sampaio y Neto (2008), que toman en cuenta los casos de los operadores de autobuses en Brasil en conjunto con varios países de Europa; $y$ de Novaes, Silveira y Medeiros (2010), relacionado con la industria de transporte autobusero de las interestatales de Brasil; la orientación fue hacia el producto en el caso de Sampaio et al. (2008), mientras que para el de Novaes et al. (2010) se empleó orientación a los insumos.

Adicional a lo anterior, con respecto a la evaluación del desempeño de los servicios que brinda el sector autobusero desde el punto de vista de corredores viales (trayecto recorrido de forma parcial o total por un conjunto de rutas en la prestación del servicio), vale señalar el trabajo desarrollado por Sheth, Triantis y Teodorovic (2007), en el cual se evaluó que ese esquema analítico propuesto puede proveer ideas operativas a las DMU sobre cómo mejorar el desempeño de la red de transporte como un todo. Ejemplo de otras investigaciones que no se centran sobre empresas son las de Barnum Hart y McNeil (2007) y Barnum, Tandon y McNeil (2008). En particular, en el estudio del 2008 se argumenta que, aunque considerar un sistema de transporte como una DMU es útil en la medida que permite comparar agencias o firmas, no lo es para evaluar actividades internas, lo cual adquiere importancia al considerar la posibilidad de la gestión multiobjetivo de las rutas, que es común en el sector autobusero.

Considerando la discusión previa, se define que la DMU sujeta de evaluación en este estudio viene dada por la ruta (concesionada o con permiso), y no por la empresa prestadora del servicio como tal; ya que una misma empresa podría prestar el servicio en diferentes rutas y, con ello, podrían compensarse los efectos en el resultado final de eficiencia de la DMU. Cabe mencionar que, para el presente caso, el análisis se centra en un corredor vial, en vez de evaluar las rutas que recorren un mismo espacio territorial. Así mismo, lo que aquí se somete a estudio y en lo que se basa la garantía de que efectivamente correspondan a DMU homogéneas, es que las rutas seleccionadas operan bajo la misma categorización definida por la Administración Pública, y, para este caso, corresponden a rutas interregionales.

9 Adicionalmente, una variación del modelo, conocida como de súpereficiencia (ver Anexo 1) permite realizar la clasificación o el ranking de las rutas eficientes, a partir de coeficientes de súpereficiencia que pueden ser iguales o superiores a la unidad. 


\section{Determinación de las variables}

Para el presente artículo fue posible obtener una base de datos a partir de una encuesta realizada al sector autobusero por parte de ARESEP en el 2009, la cual se complementó con datos de la Dirección Técnica y el Departamento de Ingeniería del Consejo de Transporte Público (CTP) ${ }^{10}$. Se seleccionan un total de 48 diferentes rutas interregionales (primer criterio de homogeneidad). Posteriormente, se procedió a definir una caracterización a nivel teórico del sector autobusero, que permitiera determinar las variables clave de insumo y de producto en el proceso de la prestación del servicio; para ello, se aplicaron entrevistas abiertas a empresarios del sector, lo que permitió contextualizar las fases que posibilitan la prestación del servicio, tanto las necesarias para iniciar la actividad (por ejemplo, el cumplimiento de las condiciones requeridas por parte de las autoridades), como aquellas para la prestación óptima diaria del servicio.

Las variables de insumo finalmente definidas se muestran en el cuadro 1, así como la manera de agruparlas con criterios que se puntualizan a continuación. Cada insumo descrito corresponde al gasto promedio realizado mensualmente por el operador para la prestación del servicio de la ruta durante el período en el que ARESEP realizó la encuesta.

CUADRO 1

DEFINICIÓN DE VARIABLES DE INSUMO

\begin{tabular}{lll}
\hline Variable & Descripción ${ }^{1}$ & Componentes \\
\hline AdmyPers & Gastos administrativos y personal & Gasto administrativo \\
RepuestyAcc & Gastos en repuestos y accesorios & Gasto en repuesto y accesorios \\
CostVar & Costos variables & Gasto en combustibles \\
& & Gasto en aceite de motor \\
& & Gasto en aceite de caja de cambios \\
& & Gasto de aceite de diferencial \\
& & Gasto en líquido de frenos \\
CostDep & Costos por depreciación & Gasto en llantas \\
& & Depreciación mensual de unidad \\
CostDep & Costos por rentabilidad & Depreciación mensual de instalaciones y equipo \\
& & Rentabilidad mensual por unidad \\
& & Rentabilidad mensual del capital en proveeduría \\
& Rentabilidad mensual del capital en instalaciones y equipos \\
\hline
\end{tabular}

Fuente: Elaboración propia.

Para las variables de producto, además, se consideró a priori la importancia de la medición de la eficiencia del servicio desde una perspectiva social, en concordancia con la visión de la ARESEP. Así, las variables de producto definidas son: promedio de pasajeros mensual por kilómetro recorrido $(\mathrm{pXkm})$ y pasajeros por bus por mes (PasBusMes).

Además, la utilización de ambas variables se prefiere sobre el uso de solo una de ellas, salvo que los resultados de los modelos estimados muestren lo contrario. La justificación de esto es que se desea un análisis en términos de eficiencia percibida por parte del usuario y suministrada por el operador, lo cual persigue múltiples objetivos. Por un lado, debe maximizar la cantidad de pasajeros que se transportan por kilómetro recorrido, de tal forma que la operación de la ruta sea económicamente viable; sin embargo, dicha maximización debe ser acorde con la capacidad permitida de los autobuses, es decir, que cumpla con la legislación vigente en la materia. Es así como la variable PasBusMes es una restricción adicional en el proceso optimizador.

10 El CTP fue creado mediante la Ley No. 7969 del 2000, Ley Reguladora del Servicio Público del Transporte Remunerado de Personas en Vehículos en la Modalidad Taxi, como un órgano de desconcentración máxima, adscrito al Ministerio de Obras Públicas y Transportes, especializado en materia de transporte público y encargado de definir las políticas y ejecutar los planes y programas nacionales relacionados con las materias de su competencia. 
Definidas las variables, se realizó un análisis de valores atípicos con el fin de excluir aquellas observaciones que presenten una desviación no aceptable por variable (segundo criterio de homogeneidad). Para ello, se empleó una batería de métodos tradicionalmente utilizados para identificación de observaciones atípicas: método gráfico, método de Wilson, súpereficiencia, pruebas estándar de distancia puntuación para outliers, criterio de distancia de Mahalanobis, $y$ criterio de fronteras parciales (de orden $m$ y alfa) (Bogetoft $y$ Otto, 2015; Daouia y Laurent, 2015; Komsta, 2015; $y$ Wilson, 2008). Del proceso se obtuvo una muestra final de 29 rutas.

Una vez detectadas y removidas las rutas con valores outliers en alguna o varias variables, se procedió a validar que estas fueran consistentes con lo definido teóricamente, por medio de técnicas exploratorias de datos. Se utilizó para ello, análisis de correlaciones (bivariadas y parciales) y pruebas de multicolinealidad, por medio de Factorización $Q R$ (Hong \& Pan, 1992) y la distancia de Mahalanobis (Penny, 1996).

Posteriormente, para la escogencia de la versión final del modelo, se procedió a la especificación de un conjunto de modelos posibles que permitiese, por medio de un análisis de sensibilidad, valorar la robustez de los resultados (similitud medida por medio de correlaciones de Pearson de las estimaciones entre coeficientes de eficiencia para una misma ruta), dada la variación en las variables seleccionadas y el supuesto sobre los rendimientos a escala. Esto se hace con el fin de evaluar, como se señaló previamente, el tipo de rendimiento a escala que debe ser asumido sobre las rutas analizadas, dado que de la revisión bibliográfica no se evidencia que haya un sobre el tipo que aplica al sector autobusero (Hidalgo \& Navarro, 2016). Tal como se aprecia en el cuadro 2 , se definen seis variaciones de modelo para estimación, en donde el primer criterio de diferenciación es el supuesto de rendimientos a escala constantes (DEA-CCR) y rendimientos a escala variables (DEA-BCC), mientras que el segundo consiste en definir las variables de producto por utilizar.

CUADRO 2

ESTRUCTURA DE LOS MODELOS CALCULADOS

\begin{tabular}{|c|c|c|c|}
\hline $\begin{array}{l}\text { Rendimientos } \\
\text { constantes }\end{array}$ & $\begin{array}{l}\text { Rendimientos } \\
\text { variables }\end{array}$ & Productos & Insumos* \\
\hline Modelo1 & Modelo 4 & $\begin{array}{l}\text { PasBusMes } \\
\text { pXkm }\end{array}$ & \multirow{3}{*}{$\begin{array}{c}\text { AdmyPers } \\
\text { RepuestyAcc } \\
\text { CostVar } \\
\text { CostRent } \\
\text { CostDep }\end{array}$} \\
\hline Modelo 2 & Modelo 5 & $p X k m$ & \\
\hline Modelo 3 & Modelo 6 & PasBusMes & \\
\hline
\end{tabular}

Fuente: Elaboración propia.

* Los insumos se aplican a todos los modelos

Asimismo, por medio de los coeficientes de intensidad $\lambda$, se determina el tipo de rendimiento a escala que presentan las rutas evaluadas. En caso de no ser posible distinguir un comportamiento marcado en torno al tipo de rendimiento, se asume que las rutas presentan rendimientos variables a escala, $y$ de esa forma se consideran factores que podrían implícitamente estar afectando de forma diferenciada los resultados de las rutas.

Tomando en consideración estos criterios, se escogió como modelo de análisis el modelo 4, que se distingue de los demás (cuadro 2) por suponer rendimientos variables a escala, $y$ la utilización de ambas variables de producto.

Por último, utilizando el modelo 4, se procedió a aplicar un modelo Tobit para determinar la significancia estadística de variables no controlables por parte de las unidades tomadoras de decisión, que previamente fueron definidas por la Administración Pública y que son fundamentales en el proceso de prestación del servicio y, por ende, influyen en la precepción que puede tener el usuario sobre este. Para este artículo, se definieron como variables de este tipo el número de carreras realizadas y la frecuencia mínima autorizada promedio de salida de una unidad de la terminal diariamente, aunque ambas resultaron ser no significativas (por tanto, se excluyen). Cabe señalar que, para el modelo finalmente seleccionado, se calcula el índice de eficiencia de escala para cada DMU.

\section{RESULTADOS}

Esta sección se divide en dos apartados. El primero resume los resultados generales del modelo que corresponden al cálculo de los coeficientes de eficiencia y la clasificación de las rutas desde la más eficiente a la menos, así como el índice de eficiencia de escala. El segundo corresponde a una aplicación de los resultados del modelo para identificar factores de mejora para las rutas ineficientes. Se recuerda que el modelo DEA utilizado (modelo 4) es la última versión de una serie de modelos calculados, que se diferencian por las variables de producto incluidas y el supuesto sobre los rendimientos a escala. 
Coeficientes de eficiencia y clasificación de las rutas

El primer resultado que se obtiene del DEA consiste en los coeficientes de eficiencia, los cuales permiten diferenciar a aquellas rutas que tuvieron un mejor desempeño en la asignación de sus recursos dado el nivel de producto que obtenían, al igual que aquellas que se desempeñaron de forma ineficiente y la distancia de ineficiencia que existe entre unas y otras. En el cuadro 3 se clasifican las 29 rutas evaluadas, ordenándolas según sus coeficientes de eficiencia y de súpereficiencia.

CUADRO 3

CLASIFICACIÓN DE LAS RUTAS

(COEFICIENTE DE EFICIENCIA Y SÚPEREFICIENCIA)

\begin{tabular}{|c|c|c|c|}
\hline Posición & No. de ruta & Eficiencia & Súpereficiencia \\
\hline 1 & $\mathrm{R} 12$ & 1,00 & 4,56 \\
\hline 2 & R42 & 1,00 & 2,79 \\
\hline 3 & R34 & 1,00 & 2,04 \\
\hline 4 & R4 & 1,00 & 1,63 \\
\hline 5 & R43 & 1,00 & 1,62 \\
\hline 6 & R29 & 1,00 & 1,60 \\
\hline 7 & $\mathrm{R} 23$ & 1,00 & 1,22 \\
\hline 8 & $\mathrm{R} 24$ & 1,00 & 1,17 \\
\hline 9 & R22 & 1,00 & 1,02 \\
\hline 10 & $\mathrm{R} 6$ & 1,00 & 1,00 \\
\hline 11 & $\mathrm{R} 2$ & 0,77 & 0,77 \\
\hline 12 & R33 & 0,76 & 0,76 \\
\hline 13 & R14 & 0,67 & 0,67 \\
\hline 14 & $\mathrm{R} 1$ & 0,54 & 0,54 \\
\hline 15 & $\mathrm{R} 17$ & 0,53 & 0,53 \\
\hline 16 & $\mathrm{R} 7$ & 0,50 & 0,50 \\
\hline 17 & R18 & 0,50 & 0,50 \\
\hline 18 & R39 & 0,50 & 0,50 \\
\hline 19 & R37 & 0,50 & 0,50 \\
\hline 20 & $\mathrm{R} 27$ & 0,49 & 0,49 \\
\hline 21 & R38 & 0,45 & 0,45 \\
\hline 22 & R36 & 0,45 & 0,45 \\
\hline 23 & R31 & 0,45 & 0,45 \\
\hline 24 & $\mathrm{R} 20$ & 0,43 & 0,43 \\
\hline 25 & $\mathrm{R} 19$ & 0,42 & 0,42 \\
\hline 26 & R3 & 0,32 & 0,32 \\
\hline 27 & $\mathrm{R} 10$ & 0,32 & 0,32 \\
\hline- & R9 & 1,00 & - \\
\hline- & R15 & 1,00 & - \\
\hline Promedio & & 0,71 & \\
\hline Desviación estándar & & 0,27 & \\
\hline
\end{tabular}

Fuente: Elaboración propia. 
De acuerdo con los resultados, 12 de las 29 rutas evaluadas se encuentran sobre la frontera de producción, es decir, dado el nivel de producto del promedio de pasajeros por kilómetro recorrido y pasajeros por bus por mes, estas rutas consumieron la menor cantidad de insumos. El caso contrario se observa para las clasificadas entre los puestos 14 y 27, cuyos coeficientes de eficiencia se encuentran por debajo de 0,55. De acuerdo con el modelo, estas rutas pueden reducir los insumos, en promedio, hasta en un $45 \%$ y seguir manteniendo el nivel de su producto.

Es importante recordar que los resultados de este modelo son sobre la eficiencia relativa, por lo que, inicialmente, la introducción de otras unidades de análisis (ya sea que muestren una mejor asignación de insumos por producto o una peor que el resto de la muestra) podría cambiar los resultados obtenidos por el modelo. De igual forma, aquellas rutas que forman la frontera empírica pueden no haber reducido en su totalidad el nivel de consumo de recursos dado el producto (frontera teórica), por lo que una mejora de sus actividades internas, ceteris paribus, ampliaría la distancia de las DMU ineficientes (en cuanto a su asignación de recursos) respecto de la frontera.

La última columna del cuadro 3 presenta, además, los resultados del modelo de súpereficiencia, los cuales permiten clasificar aquellas rutas que forman parte de la frontera empírica. Esto, a partir de un coeficiente de súpereficiencia $\theta_{i, S E}^{*} \in[0, \infty)$, el cual conforme mayor sea el valor obtenido, más "súpereficiente" será"1 ${ }^{11}$ Como se aprecia en el cuadro 3, las rutas más eficientes responden a las denominadas como R12, R42, R34, R4, R43, R29, R23, R24, R22 y R6 en ese orden respectivo, mientras que las rutas R9 y R15 se clasifican como no comparables.

\section{Ajustes de mejora}

Además de la clasificación, el modelo DEA permite identificar los ajustes que deben realizar las rutas clasificadas como ineficientes, para acercarse a la frontera empírica, de acuerdo a los parámetros del modelo. El primer ajuste que deben realizar las rutas ineficientes es a partir del cambio radial: si una ruta $i$ obtiene un coeficiente de eficiencia $0<\theta_{i}^{*}<1$; entonces, esta debe tener la posibilidad de reducir los insumos (vector $x_{i}$ ) asignados en el monto $\left(1-\theta_{i}^{*}\right) * x_{i}$ y seguir obteniendo el mismo nivel de producto. Este movimiento hace que la ruta ineficiente se mueva hacia la frontera empírica de eficiencia. Sumado a esto, se obtienen cambios adicionales a partir de las holguras en el proceso de optimización lineal que las rutas pueden realizar y seguir manteniendo el mismo nivel de eficiencia (representa un movimiento sobre la frontera empírica).

Los resultados de ajuste del modelo DEA se obtienen por separado para cada una de las rutas implicadas en la muestra. Por eso, a continuación, solo se presenta el caso ilustrativo de la ruta R2, cuyos resultados principales se muestran en el cuadro 4 (los resultados generales para el resto de las rutas ineficientes se presentan en el Anexo 2).

La ruta R2 presentó un coeficiente de eficiencia de $\theta^{*}{ }_{R 2}=0,77$, lo cual implica que, en términos relativos, se encuentra aproximadamente a un $23 \%$ de lograr su potencial en el uso de recursos, dado el nivel de producto. Como el enfoque del modelo es orientado a los insumos, la DMU de la ruta debe reducir el consumo de todos sus insumos en al menos la proporción mencionada y mantener el mismo nivel del producto, para poder alcanzar combinaciones de insumo y producto similares a las mejores dentro de la muestra. En consecuencia, para ubicarse en la frontera, la ruta R2 deberá reducir mensualmente el gasto administrativo y de personal (AdmyPers) en aproximadamente 2,23 millones de colones corrientes, el gasto de repuestos y accesorios (RepuestyAcc) en 0,98 millones, los costos variables (CostVar) en 6,69 millones, los costos por depreciación (CostDep) en 0,17 millones y el costo por rentabilidades (CostRent) en 0,57 millones y seguir manteniendo el mismo nivel de producto.

11 El proceso de optimización del modelo de súpereficiencia puede no converger para varias de las DMU analizadas antes consideradas eficientes bajos el modelo tradicional. En este caso se considera que estas opciones no son necesariamente comparables con el resto del conjunto. 
CUADRO 4

REQUERIMIENTOS DE AJUSTE PARA RUTA R2

\begin{tabular}{lcccc}
\hline \multicolumn{1}{c}{ Variable } & $\begin{array}{c}\text { Valor } \\
\text { observado }\end{array}$ & $\begin{array}{c}\text { Movimiento } \\
\text { radial }\end{array}$ & $\begin{array}{c}\text { Cambio por } \\
\text { holgura }\end{array}$ & $\begin{array}{c}\text { Valor } \\
\text { deseado }\end{array}$ \\
\hline AdmyPers $^{1}$ & 9,64 & $-2,23$ & $-1,67$ & 5,74 \\
RepuestyAcc $^{1}$ & 4,24 & $-0,98$ & $-0,72$ & 2,54 \\
CostVar $^{1}$ & 28,95 & $-6,69$ & 0,00 & 23,58 \\
CostDep $^{1}$ & 0,75 & $-0,17$ & $-0,05$ & 0,53 \\
CostRen $^{\mathrm{t} 1}$ & 2,47 & $-0,57$ & $-0,67$ & 1,23 \\
pXkm & 0,29 & 0,00 & 0,00 & 0,29 \\
PasBusMes $^{1}$ & $5.201,9$ & 0,00 & 0,00 & $5.201,9$ \\
\hline
\end{tabular}

Nota: 1. Variables en millones de colones corrientes

Fuente: Elaboración propia.

Junto a ello, a partir del cambio por holguras, el operador de la ruta puede reducir de forma adicional sus gastos mensuales en administración y personal en aproximadamente 1,67 millones de colones, en repuestos $y$ accesorios en 0,72 millones, los costos por depreciación en 0,05 millones y los costos por rentabilidad en 0,67 millones. De esta forma, dadas las condiciones en el momento de la evaluación, la ruta R2 mostraría su mejor rendimiento relativo si disminuye su gasto en administración y personal y costos variables (los dos rubros en los que más gasta), en 3,9 millones de colones y 6,69 millones de colones, respectivamente, como promedio mensual; $y$ similarmente en los gasto en repuestos y accesorios, costos por depreciación y costos por rentabilidad en los montos respectivos aproximados de $980.000,170.000$ y 570.000 colones; $y$ aun así seguir manteniendo el mismo nivel de producto.

A partir del modelo, los ajustes obtenidos resultan factibles en el tanto otras rutas comparables manejan relaciones en el uso de insumos y los productos obtenidos similares. Esto porque, del proceso de optimización, se obtiene una combinación lineal de rutas que se desempeñan mejor que la ruta analizada (aquellas cuyos valores de $\lambda$ asociados fueron iguales o superiores a 0 ), a las que se les denomina pares de comparación ${ }^{12}$. Para el caso aquí analizado, las rutas pares son las denominadas ruta $\operatorname{R15}(\lambda=0,05)$ y $\operatorname{R} 42(\lambda=0,95)$, pues de la combinación de los conjuntos de insumos $y$ de producto de ambas rutas en la proporción del correspondiente multiplicador, se obtienen los ajustes necesarios para que esta ruta R2 obtenga el rendimiento ideal, de acuerdo con las prácticas observadas en la encuesta (los pares de comparación para el resto de rutas se presentan en el Anexo 3). Con respecto a la aplicación directa, esto le permite a la ruta evaluada identificar cuáles de las otras rutas analizadas son las que mejor se le comparan y en qué proporción, de forma que puedan referenciarse en el caso particular de una o varias de estas.

Con respecto a las rutas interregionales como conjunto, en el cuadro 5 se aprecia el ajuste promedio que se debe realizar por variable evaluada, tanto absoluto como relativo. De acuerdo con esto, se observa que, aunque en términos absolutos los costos variables y los costos administrativos y de personal son aquellos en los que, en promedio, deben darse los ajustes mayores, el cambio relativo no es tan alto, pues estos son los rubros en que los operadores deben gastar más, dado que la prestación de este servicio es intensiva en su uso. En su lugar, es el gasto por rentabilidades en el que los operadores deben reducir los gastos actuales en mayor proporción para alcanzar rendimientos como los mejores operadores dentro de la muestra.

12 El resultado de la combinación lineal de la asignación de recursos y del producto obtenido de los pares de comparación (ponderados por su correspondiente $\lambda$ ) es equivalente al cambio final requerido por parte de las rutas ineficientes obtenido a través de los ajustes radiales y de holgura a partir de las variables para dichas rutas. 
CUADRO 5

AJUSTE PROMEDIO DEL SECTOR AUTOBUSERO PARA

LA RUTAS INTERREGIONALES SEGÚN VARIABLE ${ }^{1}$

\begin{tabular}{|c|c|c|c|c|}
\hline Variable & $\begin{array}{l}\text { Cambio } \\
\text { promedio }\end{array}$ & absoluto & $\begin{array}{l}\text { Cambio } \\
\text { promedio }\end{array}$ & porcentual \\
\hline AdmyPers $^{2}$ & & $-2.854,72$ & & $-32,46$ \\
\hline RepuestyAcc $^{2}$ & & $-1.221,17$ & & $-32,47$ \\
\hline CostVar ${ }^{2}$ & & $-8.799,16$ & & $-32,08$ \\
\hline CostDep ${ }^{2}$ & & $-324,14$ & & $-30,15$ \\
\hline CostRen ${ }^{2}$ & & $-1.393,92$ & & $-44,95$ \\
\hline$p X k m$ & & 0,04 & & 81,82 \\
\hline PasBusMes & & 465,91 & & 32,65 \\
\hline
\end{tabular}

Nota: 1. El cambio promedio se calcula con las 29 rutas de la muestra, tomando en cuenta el cambio radial y el cambio por holguras. 2.Valor de la variable es en miles de colones corrientes.

Fuente: Elaboración propia.

Aunque no es el caso de lo ilustrado y presentado en el cuadro 4, con la ruta R2, en ciertas rutas ineficientes, tras los ajustes de insumos, el nivel de producción de al menos uno de los insumos se puede mejorar, manteniendo la asignación de los insumos constantes, lo que se refleja en los ajustes de producto correspondientes. A ello se adiciona aquellas rutas ya eficientes dentro de la muestra que pueden, en mayor o menor proporción, aumentar su producto sin afectar el uso actual de sus recursos (súpereficientes).

Seguidamente, se muestran los resultados del índice de eficiencia de escala para cada una de las DMU que, al relacionar un modelo DEA-CCR con su equivalente DEA-BCC (el referente son las variables insumo-producto utilizados), por medio de una razón aritmética, permite identificar cuánto del nivel de eficiencia técnica relativa se debe a la especificación sobre los rendimientos de escala. A manera de ejemplo, si se toma el caso de la ruta R1, el coeficiente obtenido de comparar el modelo 4 con su equivalente DEA-CCR es de 0,9640 (modelo 1/4, según el cuadro 6). Por consiguiente, un $3,6 \%$ de la eficiencia que se obtiene en la ruta $\mathrm{R} 1$ al evaluarla con el modelo 1 se explica con el modelo 4, por la especificación de los rendimientos a escala. A nivel de aplicación, para darse una mejora en su eficiencia relativa, esta ruta debería ajustar el tamaño de su planta en esa proporción, lo que implica modificar la capacidad de activos de capital, tanto físico como humano, que intervienen en el proceso productivo de la prestación de servicio de la ruta. Un razonamiento análogo puede hacerse para las demás rutas y pares de modelos incluidos en el Cuadro 6. 
CUADRO 6

EFICIENCIA DE ESCALA DE LOS MODELOS APLICADOS

\begin{tabular}{|c|c|c|c|}
\hline No. de ruta $\backslash$ Razón modelos & Modelos 1/4 & Modelos 2/5 & Modelos 3/6 \\
\hline $\mathrm{R} 1$ & 0,9640 & 0,7623 & 0,8688 \\
\hline $\mathrm{R} 2$ & 0,8833 & 0,8262 & 0,8815 \\
\hline R3 & 0,4749 & 0,3354 & 0,4749 \\
\hline R4 & 1,0000 & 0,9909 & 1,0000 \\
\hline R6 & 0,2426 & 0,0464 & 0,2426 \\
\hline R7 & 0,2722 & 0,2520 & 0,2675 \\
\hline R9 & 0,7210 & 0,6909 & 0,8089 \\
\hline $\mathrm{R} 10$ & 0,5455 & 0,4088 & 0,5455 \\
\hline $\mathrm{R} 12$ & 1,0000 & 1,0000 & 1,0000 \\
\hline R14 & 0,7466 & 0,4652 & 0,7466 \\
\hline R15 & 0,5780 & 0,8774 & 0,5780 \\
\hline R17 & 0,2656 & 0,2383 & 0,2656 \\
\hline $\mathrm{R} 18$ & 0,3627 & 0,2907 & 0,3627 \\
\hline R19 & 0,7020 & 0,2907 & 0,7020 \\
\hline $\mathrm{R} 20$ & 0,9708 & 0,7199 & 0,9708 \\
\hline $\mathrm{R} 22$ & 0,1614 & 0,1196 & 0,1614 \\
\hline $\mathrm{R} 23$ & 1,0000 & 0,8750 & 1,0000 \\
\hline R24 & 1,0000 & 1,0000 & 1,0000 \\
\hline $\mathrm{R} 27$ & 0,6044 & 0,5757 & 0,5252 \\
\hline R29 & 1,0000 & 1,0000 & 0,7741 \\
\hline R31 & 0,9987 & 0,7258 & 0,9987 \\
\hline R33 & 0,9649 & 0,6869 & 0,9821 \\
\hline R34 & 1,0000 & 1,0000 & 0,7741 \\
\hline R36 & 0,4875 & 0,3785 & 0,4875 \\
\hline R37 & 0,9979 & 0,7641 & 0,9979 \\
\hline R38 & 0,9927 & 0,8409 & 0,9988 \\
\hline R39 & 0,6521 & 0,5548 & 0,6521 \\
\hline R42 & 1,0000 & 0,9147 & 0,9975 \\
\hline R43 & 0,5930 & 0,3532 & 0,5930 \\
\hline
\end{tabular}

Fuente: Elaboración propia.

\section{Aplicaciones del modelo a la regulación}

Desde un esquema de regulación basado en incentivos, se tiene como objetivo promover la eficiencia entre los operadores, para los cuales puede ser de interés implementar evaluaciones comparativas de eficiencia entre empresas, como un parámetro para promover la competencia por comparación (benchmarking) (Coelli, Estache, Perelman, \& Trujillo, 2003). Las líneas de acción más utilizadas por reguladores de servicios públicos para definir los parámetros de productividad con los cuales se realiza el benchmarking regulatorio son dos. La primera es la comparación de productividades para los operadores regulados con tecnología y procesos productivos similares. Posteriormente, partiendo de que se mantiene cierta estabilidad del entorno, el regulador debería esperar que el rendimiento del operador con el paso de los años al menos se mantenga, con eventuales manifestaciones de 
mejora. Por ello, se analiza la evolución de productividades del operador regulado en el tiempo, tomando como referencia, además, las condiciones promedio del sector o del operador con mejor rendimiento relativo dentro del grupo de comparación. En algunos casos se suelen definir, de forma adicional, metas para los operadores como elemento de incentivos para la regulación (tradicionalmente llamado "factor $X$ ").

Para el cálculo de los parámetros de productividad se suele trabajar, con índices (los cuales utilizan precios de mercado) o con modelos de frontera como el DEA y el SFA ${ }^{13}$, cuyos coeficientes estimados funcionan como "precios sombra" para los insumos y los productos.

Específicamente, en el modelo regulatorio vigente de transporte público en Costa Rica, la asignación tarifaria se compone de un conjunto de rubros de gasto necesarios para la prestación del servicio, en los cuales se basó la información utilizada para el cálculo del modelo DEA en apartados previos. No obstante, tal como afirman Coelli et al. (2003), el regulador comúnmente querrá tomar en cuenta los factores de ineficiencia asignativa de recursos en los que incurre el operador regulado para la definición del "factor X" (en este caso, la tarifa en la prestación del servicio). Esto no se refleja en el modelo tarifario vigente, en donde el reporte de los gastos incurridos por los operadoradores no diferencia el redimiento relativo que tuvieron en la asignación de estos, es decir, indirectamente este modelo parte de que los operadores son igual de eficientes en la aplicación de los recursos.

Bajo esta premisa, los autores de este documento consideran relevante la implementación de herramientas como la expuesta anteriormente como parte de un esquema de regulación. En este caso en particular, el regulador tiene la posibilidad, al comparar un conjunto de rutas homogéneas ${ }^{14}$, de definir parámetros mínimos de rendimiento relativo para un grupo en particular, en donde se pueda penalizar aquellos a los que -pese a reportar cierto nivel de gasto en insumos-, no presentan el rendimiento mínimo deseado en la aplicación de recursos para la prestación del servicio, según las prácticas observada del servicio prestado en otras rutas, que se toman como comparación.

Adicionalmente, como se aprecia en el apartado de ajustes de mejora, la metodología no solo le permite la clasificación de las rutas, sino identificar las áreas, según los aspectos que el regulador consideró relevante evaluar, que generan el bajo rendimiento relativo del operador en la prestación de la ruta evaluada y en qué cuantía; de forma que se pueden indicar estos factores de ajuste a los operadores. Es claro que en la aplicación de medidas correctivas, el regulador debe tomar en cuenta aspectos que en ocasiones solo pueden verificarse a partir del análisis detallado de cada caso, y que pueden limitar la reducción inmediata de los insumos ${ }^{15}$. A pesar de ello, los resultados que obtenga el regulador deben fungir en un primer momento como una señal de "alerta", que llame la atención a este respecto del rendimiento que el operador está obteniendo, así como para evaluar si este último trabaja en función de los mismos objetivos que busca el regulador (Bonifaz y Santín, 2000).

Cuando exista información homogénea para las rutas del sector, en dos años o más, a partir de los indicadores de eficiencia, se pueden calcular indicadores de productividad que le permitan al regulador evaluar la evolución individual de un operador a través del tiempo. Esto potencia que pueda identificar metas con el resto del sector, no solo de plazo inmediato, sino también en el largo plazo, tanto individuales como colectivas.

Es importante recordar que la aplicación de mecanismos sofisticados, como este y otros modelos de frontera, requiere de la mejor información disponible presentada por las empresas reguladas; de manera que, conforme la información sea más detallada, veraz y homogénea dentro las rutas evaluadas, mejor y más confiable serán los resultados y el análisis obtenidos para efectos del proceso de toma de decisiones en la materia de regulación. Tal vez el instrumento esencial para potenciar esto es la implementación de esquemas de contabilidad regulatoria que contengan como rubros información para generar las variables de interés.

13 El análisis de frontera estocástica (SFA por sus siglas en inglés), es una alternativa paramétrica a los modelos de frontera, el cual, en contraste con el DEA, requiere una especificación a priori de la función de producción, pero contrario al modelo de optimización, puede tomar en cuenta el ruido en los datos y la aplicación de pruebas de hipótesis.

14 Esto en sí mismo implica, para el regulador, un trabajo arduo en la definición de las rutas, de forma que establezca, antes de evaluar los casos extremos u outliers, las características relevantes para cada grupo de comparación, de manera que este grupo de comparación sea el más consistente y comparable en función de las carácterísticas del sector y de los aspectos en los que se considera el operador de rutas debe ser eficiente.

15 Ejemplos pueden ser las limitaciones contractuales o estructurales que tienen en ese momento los operadores, por rigideces de corto plazo entre la relación de los insumos con el capital existente. 


\section{CONCLUSIONES}

En los apartados previos desarrollados en este documento, se da un acercamiento a la aplicación de modelos de frontera para la evaluación del transporte público en modalidad de autobús; siendo esta la primera aplicación, para el caso costarricense, de este tipo de herramientas de análisis al sector. En este trabajo, se utilizó un modelo DEA-BCC, enfocado a los insumos, a 29 rutas interregionales distribuidas a nivel nacional, tras un proceso previo de eliminación de rutas consideradas como casos extremos u outliers.

De esta forma, a partir del modelo, se pudo calcular los coeficientes de eficiencia relativa de las rutas en cuestión, de las cuales 12 son eficientes of forman parte de la frontera empírica. Pese a ello, se aprecia que cerca del $48 \%$ de las rutas evaluadas mantienen niveles de eficiencia muy bajos (por debajo de 0,55), lo cual muestra una diferencia importante entre las rutas evaluadas dentro del conjunto de rutas interregionales. Adicionalmente, se pudo calcular los factores de mejora para las rutas ineficientes, lo cual sirve como guía para que estas puedan identificar aquellos insumos en los que, según se reporta, realizan un gasto excesivo que no se refleja en los insumos mostrados.

En consecuencia, con lo descrito al inicio del artículo, la principal pretensión de este era proporcionar una metodología de análisis del sector de transporte público, a nivel de prestación del servicio por ruta concesionada, que pudiera ilustrar la aplicabilidad y utilidad de este tipo de instrumentos para el proceso de toma de decisiones a nivel de regulación; en donde el modelo regulatorio actual no diferencia a los operadores regulados según qué tan eficiente ha sido la asignación de recursos utilizados para la prestación del servicio (lo cual no es igual para las rutas interregionales, según los resultados de este estudio).

Dentro de esa misma línea, se destaca la importancia del debate de implementación de mecanismos de evaluación de la eficiencia para el análisis y la regulación del transporte público y de otros sectores regulados por el Estado. También se reafirma la necesidad de mejorar las fuentes de información que las autoridades puedan obtener, en función de lograr resultados más concretos y fidedignos de los objetivos que este desea se optimicen a nivel del sector regulado.

\section{AGRADECIMIENTOS}

Se les agradece a los funcionarios de ARESEP y CTP por proporcionar información para el desarrollo de investigación, al igual que a los profesores Yanira Xirinachs-Salázar, Max Soto Jiménez y Marlon Yong Chacón por los comentarios realizados para el trabajo previo del que se deriva este artículo. 


\section{REFERENCIAS}

Andersen, P., \& Petersen, N. (1993). A procedure for ranking efficient units in data envelopment analysis. Management Science, 39(10), 1261-1264.

Barnum, D., Hart, J., y McNeil, S. (2007). Comparing the Efficiency of Public Transportation Subunits Using Data Envelope Analysis. Journal of Public Transportation, 10(2), 1-16. https://doi.org/10.5038/23750901.10.2.1

Barnum, D. T., Tandon, S., \& McNeil, S. (2008). Comparing the Performance of Bus Routes after Adjusting for the Environment Using Data Envelopment Analysis. Journal of Transportation Engineering, 134(2), 77-85. https://doi.org/10.1061/(ASCE)0733-947X(2008)134:2(77)

Bogetoft, P., y Otto, L. (2015). Benchmark and Frontier Analysis Using DEA and SFA. Viena, Austria: CRAN-Project. Recuperado de https://cran.r-project.org/web/packages/Benchmarking/Benchmarking.pdf

Bonifaz F., J. L., \& Santin, D. (2000). Eficiencia relativa de las empresas distribuidoras de energía eléctrica en el Perú: una aplicación del análisis envolvente de datos (DEA). Apuntes: Revista de Ciencias Sociales, (47), 111-138. https://doi.org/10.21678/apuntes.47.499

Charnes, A., Cooper, W. W., \& Rhodes, E. (1978). Measuring the efficiency of decision making units. European Journal of Operational Research, 2(6), 429-444. https://doi.org/10.1016/0377-2217(78)90138-8

Coelli, T., Estache, A., Perelman, S., \& Trujillo, L. (2003). Una introducción a las medidas de eficiencia para reguladores de servicios públicos y de transporte. Colombia: Banco Mundial en coedición con Alfaomega Colombiana S.A.

Coelli, T., Passada, D., O'Donnell, C., \& Battese, G. (2005). An Introduction to Efficiency and Productivity Analysis ( $2^{\circ}$ ed.). Boston: Kluwer Academic.

Daouia, A., \& Laurent, T. (2015). Partial Frontier Analysis. Viena, Austria: CRAN-Project. Recuperado de https://cran.r-project.org/web/packages/frontiles/frontiles.pdf

Daraio, C., \& Simar, L. (2007). Advanced Robust and Nonparametric Methods in Efficiency Analysis. Nueva York, Estados Unidos: Springer Science+Business Media, LLC.

De Borger, B., Kerstens, K., \& Costa, Á. (2002). Public transit performance: What does one learn from frontier studies? Transport Reviews, 22(1), 1-38. https://doi.org/10.1080/01441640010020313

Farrell, M. J. (1957). The Measurement of Productive Efficiency. Journal of the Royal Statistical Society. Series A (General), 120(3), 253. https://doi.org/10.2307/2343100

Garcia, I. M. (2009). Technical and Scale Efficiency in Spanish Urban Transport: Estimating with Data Envelopment Analysis. Advances in Operations Research, 2009, 1-15. https://doi.org/10.1155/2009/721279

Hidalgo, J., \& Navarro, D. (2016). Análisis de la Eficiencia Técnica del Sector de Transporte Público: Costa Rica 2009. (Tesis para optar por el grado de Licenciatura), San José, Costa Rica, Universidad de Costa Rica.

Hong, Y. P., \& Pan, C.-T. (1992). Rank-Revealing QR Factorizations and the Singular Value Decomposition. Mathematics of Computation, 58(197), 213-232. https://doi.org/10.2307/2153029

Karlaftis, M., Gleason, J., y Barnum, D. (2013). Bibliography of Urban Transit Data Envelopment Analysis (DEA) Publications. Recuperado de https://papers.ssrn.com/sol3/papers.cfm?abstract_id=1350583

Komsta, L. (2015). Tests for Outliers. Viena, Austria: CRAN-Project. Recuperado de https://cran.r-project. org/web/packages/outliers/outliers.pdf

LCR Logística. (2002). Consultoría para la elaboración de una encuesta sobre los operadores del transporte reminerado de personas, modalidad autobús, en todo el país. Informe Final de la licitación restringida No. I-2001 de ARESEP [documento no publicado], San José, Costa Rica.

Lovell, C. A. K., \& Rouse, A. P. B. (2003). Equivalent standard DEA models to provide super-efficiency sco- 
res. Journal of the Operational Research Society, 54(1), 101-108. https://doi.org/10.1057/palgrave.jors.2601483

Novaes, A. G. N., Silveira, S. F., \& Medeiros, H. C. (2010). Efficiency and productivity analysis of the interstate bus transportation industry in Brazil. Pesquisa Operacional, 30(2), 465-485. https://doi.org/10.1590/ S0101-74382010000200012

Penny, K. (1996). Appropriate Critical Values When Testing for a Single Multivariate Outlier by Using the Mahalanobis Distance. Journal of the Royal Statistical Society. Series C (Applied Statistics), 45(1), 73-81. https:// doi.org/10.2307/2986224

Sampaio, B. R., Neto, O. L., \& Sampaio, Y. (2008). Efficiency analysis of public transport systems: Lessons for institutional planning. Transportation Research Part A: Policy and Practice, 42(3), 445-454. https://doi. org/10.1016/j.tra.2008.01.006

Sheth, C., Triantis, K., \& Teodorovi , D. (2007). Performance evaluation of bus routes: A provider and passenger perspective. Transportation Research Part E: Logistics and Transportation Review, 43(4), 453-478. https:// doi.org/10.1016/j.tre.2005.09.010

Wilson, P. W. (2008). FEAR: A software package for frontier efficiency analysis with R. Socio-Economic Planning Sciences, 42(4), 247-254. https://doi.org/10.1016/j.seps.2007.02.001 
VII. ANEXOS

\section{Anexo 1}

Modelo DEA de súpereficiencia

El modelo de súpereficiencia fue planteado por Andersen y Petersen (1993) como una herramienta para definir la jerarquía entre diferentes DMU que se encuentran en la frontera de producción. La esencia del modelo consiste en evaluar en indicador o coeficiente de eficiencia de una DMU específica, pero descontando la restricción propia de la unidad analizada, de forma que solo se compare con las demás DMU de la muestra.

Sea la $i$-ésima DMU, en donde se definen las matrices $Y_{i}$, de tamaño $M x(I-1)$, y $X_{i}$, de tamaño $N_{x}(I-1)$, representando respectivamente los conjuntos de producto e insumo del total de la muestra, pero descontando el conjunto de la DMU evaluada. Posteriorment $\forall i=1, \ldots, I \quad$ nización se desarrolla de la siguiente forma.

$$
\begin{gathered}
\min _{\lambda, \theta_{i}} \theta_{i, S E} \\
\text { s. } a . \\
Y_{i} \lambda-y_{i} \geq 0 \\
\theta x_{i}-X_{i} \lambda \geq 0 \\
I 1^{\prime} \mathrm{x} \lambda=1 \\
\lambda \geq 0 \\
\theta_{i, S E} \text { libre }
\end{gathered}
$$

Nótese que en contraste con el modelo DEA-BCC citado anteriormente, $\theta_{i, S E}^{*}$ es libre, pues puede crecer indefinidamente. Cabe agregar que no todos los problemas de súpereficiencia tenga solución factible, lo cual contrasta con el modelo original, en donde el valor sería $\theta_{i}^{*}=1, y$ cuya explicación implicaría que esta unidad de análisis aparenta eficiencia relativa por no ser comparable con el resto de las DMU de la muestra (Lovell \& Rouse, 2003). 
Anexo 2

Ajustes por movimiento radial y por holguras para rutas según variable

CUADRO 7

AJUSTES DE LA VARIABLE ${ }^{1}$ POR RUTA

\begin{tabular}{|c|c|c|c|c|}
\hline Ruta & $\begin{array}{l}\text { Valor de la } \\
\text { variable }\end{array}$ & $\begin{array}{l}\text { Movimiento } \\
\text { radial }\end{array}$ & $\begin{array}{l}\text { Cambio por } \\
\text { holgura }\end{array}$ & Valor deseado \\
\hline R1 & $4.828 .217,5$ & $2.201 .184,36$ & 0,00 & $2.627 .033,14$ \\
\hline $\mathrm{R} 2$ & $9.639 .072,56$ & $2.227 .589,67$ & $1.670 .411,64$ & 5.741 .071 .25 \\
\hline R3 & 8.053 .934 .68 & $5.484 .729,52$ & 0,00 & $2.569 .205,16$ \\
\hline R4 & $1.607 .512,09$ & 0,00 & 0,00 & $1.607 .512,09$ \\
\hline $\mathrm{R} 6$ & $1.606 .512,09$ & 0,00 & 0,00 & $1.606 .512,09$ \\
\hline R7 & $4.845 .775,91$ & $2.395 .515,16$ & $364.87,74$ & $2.065 .443,01$ \\
\hline R9 & $12.702 .779,76$ & 0,00 & 0,00 & $12.702 .779,76$ \\
\hline R10 & $8.053 .934,68$ & $5.484 .729,52$ & 0,00 & $2.569 .205,16$ \\
\hline R12 & $3.213 .070,86$ & 0,00 & 0,00 & $3.213 .070,86$ \\
\hline R14 & $4.762 .240,23$ & $1.572 .491,72$ & $375.166,25$ & $2.814 .582,25$ \\
\hline R15 & $24.119 .384,42$ & 0,00 & 0,00 & $24.119 .384,42$ \\
\hline R17 & $3.217 .183,94$ & $1.510 .467,86$ & $2.556,77$ & $1.704 .159,31$ \\
\hline R18 & $3.218 .881,67$ & $1.612 .302,76$ & 0,00 & $1.606 .508,90$ \\
\hline R19 & $8.042 .959,84$ & $4.647 .222,20$ & 0,00 & $3.395 .737,65$ \\
\hline $\mathrm{R} 20$ & $4.827 .287,37$ & $2.741 .899,23$ & 0,00 & $2.085 .388,14$ \\
\hline $\mathrm{R} 22$ & $6.362 .964,84$ & 0,00 & 0,00 & $6.362 .964,84$ \\
\hline R23 & $3.210 .896,44$ & 0,00 & 0,00 & $3.210 .896,44$ \\
\hline R24 & $1.611 .576,14$ & 0,00 & 0,00 & $1.611 .576,14$ \\
\hline R27 & $3.221 .573,87$ & $1.614 .974,98$ & 0,00 & $1.606 .598,89$ \\
\hline R29 & $1.606 .535,43$ & 0,00 & 0,00 & $1.606 .535,43$ \\
\hline R31 & $22.501 .297,39$ & $12.497 .220,57$ & $6.144 .135,21$ & $3.859 .941,61$ \\
\hline R33 & $6.429 .855,93$ & $1.516 .160,03$ & $1.059 .105,42$ & $3.854 .590,48$ \\
\hline R34 & $6.349 .746,98$ & 0,00 & 0,00 & $6.349 .746,98$ \\
\hline R36 & $6.434 .367,88$ & $3.544 .049,83$ & 0,00 & $2.890 .318,05$ \\
\hline R37 & $11.127 .086,00$ & $5.720 .434,91$ & $934.777,04$ & $4.471 .874,05$ \\
\hline R38 & $20.664 .588,28$ & $11.369 .656,47$ & $4.808 .667,21$ & $4.486 .264,60$ \\
\hline R39 & $3.221 .573,87$ & $1.614 .974,98$ & 0,00 & $1.606 .598,89$ \\
\hline R42 & $4.822 .391,94$ & 0,00 & 0,00 & 4.822.391,94 \\
\hline R43 & $12.823 .159,35$ & 0,00 & 0,00 & $12.823 .159,35$ \\
\hline
\end{tabular}

Nota: 1. Variable en colones corrientes.

Fuente: elaboración propia. 
CUADRO 8

AJUSTES DE LA VARIABLE ${ }^{1}$ POR RUTA

\begin{tabular}{|c|c|c|c|c|}
\hline Ruta & $\begin{array}{l}\text { Valor de la } \\
\text { variable }\end{array}$ & $\begin{array}{l}\text { Movimiento } \\
\text { radial }\end{array}$ & $\begin{array}{l}\text { Cambio por } \\
\text { holgura }\end{array}$ & Valor deseado \\
\hline $\mathrm{R} 1$ & $2.163 .971,25$ & $986.554,49$ & $20.525,94$ & $1.156 .890,82$ \\
\hline $\mathrm{R} 2$ & $4.242 .832,50$ & $980.518,59$ & $718.520,38$ & $2.543 .793,53$ \\
\hline $\mathrm{R} 3$ & $3.640 .469,32$ & $2.479 .159,61$ & $34.418,83$ & $1.126 .890,88$ \\
\hline $\mathrm{R} 4$ & $711.804,87$ & 0,00 & 0,00 & $711.804,87$ \\
\hline $\mathrm{R} 6$ & $707.138,75$ & 0,00 & 0,00 & $707.138,75$ \\
\hline $\mathrm{R} 7$ & $2.152 .002,66$ & $1.068 .254,12$ & $174.276,80$ & $909.471,74$ \\
\hline $\mathrm{R} 9$ & $4.925 .164,00$ & 0,00 & 0,00 & $4.925 .164,00$ \\
\hline $\mathrm{R} 10$ & $3.640 .469,32$ & $2.479 .159,61$ & $34.418,83$ & $1.126 .890,88$ \\
\hline $\mathrm{R} 12$ & $1.414 .506,29$ & 0,00 & 0,00 & $1.414 .506,29$ \\
\hline $\mathrm{R} 14$ & $1.840 .553,25$ & $607.750,68$ & 0,00 & $1.232 .802,57$ \\
\hline R15 & $10.713 .468,75$ & 0,00 & 0,00 & $10.713 .468,75$ \\
\hline $\mathrm{R} 17$ & $1.434 .668,44$ & $673.576,83$ & $7.551,85$ & $753.539,76$ \\
\hline $\mathrm{R} 18$ & $1.442 .647,50$ & $722.622,13$ & $12.799,64$ & $707.225,73$ \\
\hline $\mathrm{R} 19$ & $3.586 .671,09$ & $2.072 .378,56$ & $31.389,26$ & $1.482 .903,28$ \\
\hline $\mathrm{R} 20$ & 2.159.411,79 & $1.226 .545,89$ & $14.791,49$ & $918.074,40$ \\
\hline $\mathrm{R} 22$ & $2.519 .322,00$ & 0,00 & 0,00 & $2.519 .322,00$ \\
\hline $\mathrm{R} 23$ & $1.403 .847,35$ & 0,00 & 0,00 & $1.403 .847,35$ \\
\hline $\mathrm{R} 24$ & $731.962,50$ & 0,00 & 0,00 & $731.962,50$ \\
\hline $\mathrm{R} 27$ & $1.456 .187,73$ & $729.986,91$ & $18,922,92$ & $707.277,90$ \\
\hline $\mathrm{R} 29$ & $707.253,15$ & 0,00 & 0,00 & $707.253,15$ \\
\hline R31 & $9.949 .590,00$ & $5.526 .002,29$ & $2.725 .105,34$ & $1.698 .482,37$ \\
\hline R33 & $2.847 .219,47$ & $671.374,35$ & $509.139,13$ & 1.666.705.99 \\
\hline R34 & $2.454 .528,58$ & 0,00 & 0,00 & $2.454 .528,58$ \\
\hline R36 & $2.869 .336,88$ & $1.580 .430,75$ & $23.419,65$ & $1.265 .486,47$ \\
\hline R37 & $4.369 .095,50$ & $2.246 .152,00$ & $146.623,07$ & $1.976 .320,43$ \\
\hline R38 & $8.114 .034,50$ & $4.464 .341,78$ & $1.716 .175,60$ & $1.933 .517,12$ \\
\hline R39 & $1.456 .187,73$ & $729.986,91$ & $17,991,14$ & $708.209,68$ \\
\hline R42 & $2.135 .414,61$ & 0,00 & 0,00 & $2.135 .414,61$ \\
\hline R43 & $5.515 .260,00$ & 0,00 & 0,00 & $5.515 .260,00$ \\
\hline
\end{tabular}

Nota: 1. Variable en colones corrientes. 
Fuente: elaboración propia.

CUADRO 9

AJUSTES DE LA VARIABLE ${ }^{1}$ POR RUTA

\begin{tabular}{|c|c|c|c|c|}
\hline Ruta & $\begin{array}{l}\text { Valor de la } \\
\text { variable }\end{array}$ & $\begin{array}{l}\text { Movimiento } \\
\text { radial }\end{array}$ & $\begin{array}{c}\text { Cambio por } \\
\text { holgura }\end{array}$ & Valor deseado \\
\hline $\mathrm{R} 1$ & $16.515 .372,20$ & 7.529.358,19 & $4.305 .760,85$ & $4.680 .253,16$ \\
\hline $\mathrm{R} 2$ & 28.953.321,10 & $6.691 .112,51$ & 0,00 & $22.262 .208,59$ \\
\hline R3 & $26.983 .990,67$ & $18.376 .097,65$ & $728.011,79$ & $7.879 .881,24$ \\
\hline $\mathrm{R} 4$ & $4.517 .902,77$ & 0,00 & 0,00 & $4.517 .902,77$ \\
\hline $\mathrm{R} 6$ & $20.691 .585,14$ & 0,00 & 0,00 & $20.691 .585,14$ \\
\hline R7 & $12.149 .860,39$ & $6.031 .190,70$ & 0,00 & $6.118 .669,69$ \\
\hline R9 & $15.787 .199,42$ & 0,00 & 0,00 & $15.787 .199,42$ \\
\hline $\mathrm{R} 10$ & $25.383 .494,19$ & $17.286 .159,55$ & $217.432,94$ & $7.879 .901,71$ \\
\hline $\mathrm{R} 12$ & 3.566.097,63 & 0,00 & 0,00 & $3.566 .097,63$ \\
\hline $\mathrm{R} 14$ & $18.536 .706,72$ & $6.120 .820,56$ & $3.678 .295,10$ & $8.737 .591,06$ \\
\hline $\mathrm{R} 15$ & $83.642 .501,86$ & 0,00 & 0,00 & $83.642 .501,86$ \\
\hline $\mathrm{R} 17$ & $9.153 .384,27$ & $4.297 .513,91$ & 0,00 & $4.855 .870,35$ \\
\hline $\mathrm{R} 18$ & $9.936 .073,98$ & $4.976 .97,45$ & 0,00 & $4.959 .094,52$ \\
\hline R19 & $25.884 .872,08$ & $14.956 .279,09$ & $158.584,54$ & $10.770 .008,45$ \\
\hline $\mathrm{R} 20$ & $16.020 .994,32$ & $9.099 .924,77$ & $732.452,89$ & $6.188 .616,65$ \\
\hline $\mathrm{R} 22$ & $20.691 .585,14$ & 0,00 & 0,00 & $20.691 .585,14$ \\
\hline $\mathrm{R} 23$ & $10.123 .029,69$ & 0,00 & 0,00 & $10.123 .029,69$ \\
\hline $\mathrm{R} 24$ & $1.884 .469,90$ & 0,00 & 0,00 & $1.884 .469,90$ \\
\hline $\mathrm{R} 27$ & $5.807 .593,61$ & $2.911 .346,68$ & 0,00 & $2.896 .246,93$ \\
\hline $\mathrm{R} 29$ & $2.195 .372,24$ & 0,00 & 0,00 & $2.195 .372,24$ \\
\hline R31 & $78.417 .336,07$ & $43.552 .988,45$ & $21.089 .850,67$ & $13.774 .496,95$ \\
\hline R33 & $19.193 .965,47$ & $4.525 .937,06$ & $2.905 .729,01$ & $11.762 .299,40$ \\
\hline R34 & $6.749 .133,91$ & 0,00 & 0,00 & $6.749 .133,91$ \\
\hline R36 & $20.129 .637,63$ & $11.087 .404,41$ & $39.875,51$ & $9.002 .357,72$ \\
\hline R37 & $38.536 .960,83$ & $19.811 .851,56$ & $1.503 .122,43$ & $17.221 .986,84$ \\
\hline R38 & $55.420 .969,61$ & $30.492 .617,48$ & $10.398 .620,24$ & $14.529 .731,89$ \\
\hline R39 & $9.363 .846,00$ & $4.694 .096,00$ & $1.953 .269,74$ & $2.716 .480,26$ \\
\hline $\mathrm{R} 42$ & $19.193 .965,47$ & 0,00 & 0,00 & $19.193 .965,47$ \\
\hline R43 & $43.763 .387,21$ & 0,00 & 0,00 & $43.763 .387,21$ \\
\hline
\end{tabular}

Nota: 1. Variable en colones corrientes. 
Fuente: elaboración propia.

CUADRO 10

AJUSTES DE LA VARIABLE ${ }^{1}$ POR RUTA

\begin{tabular}{|c|c|c|c|c|}
\hline Ruta & $\begin{array}{l}\text { Valor de la } \\
\text { variable }\end{array}$ & $\begin{array}{l}\text { Movimiento } \\
\text { radial }\end{array}$ & $\begin{array}{l}\text { Cambio por } \\
\text { holgura }\end{array}$ & Valor deseado \\
\hline $\mathrm{R} 1$ & $927.622,34$ & $422.903,03$ & 0,00 & $504.19,32$ \\
\hline $\mathrm{R} 2$ & $752.440,53$ & $173.889,01$ & $46.783,90$ & $531.767,62$ \\
\hline $\mathrm{R} 3$ & $1.367 .492,66$ & $931.262,50$ & 0,00 & $436.230,16$ \\
\hline $\mathrm{R} 4$ & $490.214,13$ & 0,00 & 0,00 & $490.214,13$ \\
\hline $\mathrm{R} 6$ & $710.012,20$ & 0,00 & 0,00 & $710.012,20$ \\
\hline $\mathrm{R} 7$ & $922.491,81$ & $457.924,93$ & 0,00 & $464.566,87$ \\
\hline $\mathrm{R} 9$ & $777.355,05$ & 0,00 & 0,00 & $777.355,05$ \\
\hline $\mathrm{R} 10$ & $1.367 .492,66$ & $931.262,50$ & 0,00 & $436.230,16$ \\
\hline $\mathrm{R} 12$ & $538.451,05$ & 0,00 & 0,00 & $538.451,05$ \\
\hline $\mathrm{R} 14$ & $630.735,20$ & $208.268,76$ & 0,00 & $422.466,44$ \\
\hline $\mathrm{R} 15$ & $1.021 .350,69$ & 0,00 & 0,00 & $1.021 .350,69$ \\
\hline $\mathrm{R} 17$ & $913.883,79$ & $429.068,44$ & 0,00 & $484.815,35$ \\
\hline $\mathrm{R} 18$ & $1.305 .389,90$ & $653.869,90$ & $94.661,76$ & $556.858,34$ \\
\hline R19 & $939.707,83$ & $542.963,18$ & 0,00 & $396.744,64$ \\
\hline $\mathrm{R} 20$ & $1.072 .566,61$ & $609.217,84$ & 0,00 & $463.348,78$ \\
\hline $\mathrm{R} 22$ & $345.147,11$ & 0,00 & 0,00 & $345.147,11$ \\
\hline $\mathrm{R} 23$ & $400.249,86$ & 0,00 & 0,00 & $400.249,86$ \\
\hline $\mathrm{R} 24$ & $1.315 .859,44$ & 0,00 & 0,00 & $1.315 .859,44$ \\
\hline $\mathrm{R} 27$ & $1.341 .281,28$ & $672.384,30$ & $132.084,76$ & $536.812,21$ \\
\hline R29 & $529.964,01$ & 0,00 & 0,00 & $529.964,01$ \\
\hline R31 & $997.175,66$ & $553.831,36$ & 0,00 & $443.344,30$ \\
\hline R33 & $515.839,10$ & $121.634,86$ & 0,00 & $394.204,24$ \\
\hline R34 & $481.902,01$ & 0,00 & 0,00 & $481.902,01$ \\
\hline R36 & $931.099,82$ & $512.849,78$ & 0,00 & $418.250,04$ \\
\hline R37 & $996.094,33$ & $512.092,10$ & 0,00 & $484.002,24$ \\
\hline R38 & $1.041 .033,60$ & $572.776,69$ & 0,00 & $468.256,91$ \\
\hline R39 & $1.341 .281,28$ & $672.384,30$ & $147.919,28$ & $520.977,69$ \\
\hline R42 & $507.297,44$ & 0,00 & 0,00 & $507.297,44$ \\
\hline R43 & $220.068,72$ & 0,00 & 0,00 & $\overline{220.068,72}$ \\
\hline
\end{tabular}

Nota: 1. Variable en colones corrientes. 
Fuente: elaboración propia.

CUADRO 11

AJUSTES DE LA VARIABLE ${ }^{1}$ POR RUTA

\begin{tabular}{lrrrr}
\hline Ruta & \multicolumn{1}{c}{$\begin{array}{c}\text { Valor de la } \\
\text { variable }\end{array}$} & \multicolumn{1}{c}{$\begin{array}{c}\text { Movimiento } \\
\text { radial }\end{array}$} & $\begin{array}{c}\text { Cambio por } \\
\text { holgura }\end{array}$ & \multicolumn{1}{c}{ Valor deseado } \\
\hline R1 & $1.743 .523,51$ & $794.872,37$ & $458.881,50$ & $489.769,64$ \\
R2 & $2.468 .939,86$ & $570.572,00$ & $666.673,20$ & $1.231 .694,66$ \\
R3 & $4.309 .908,96$ & $2.935 .048,00$ & 0,00 & $399.209,66$ \\
R4 & $283.896,42$ & 0,00 & 0,00 & $283.896,42$ \\
R6 & $411.489,98$ & 0,00 & 0,00 & $411.489,98$ \\
R7 & $1.730 .112,55$ & $858.827,87$ & $532.409,00$ & $338.875,68$ \\
R9 & $3.284 .088,95$ & 0,00 & 0,00 & $3.284 .088,95$ \\
R10 & $4.309 .908,96$ & $2.935 .048,00$ & $975.651,30$ & $399.209,66$ \\
R12 & $637.712,97$ & 0,00 & 0,00 & $637.712,97$ \\
R14 & $1.071 .033,39$ & $353.655,23$ & $288.704,90$ & $428.673,26$ \\
R15 & $8.833 .593,66$ & 0,00 & 0,00 & $8.833 .593,66$ \\
R17 & $1.153 .408,37$ & $541.525,23$ & $316.370,00$ & $295.513,14$ \\
R18 & $1.706 .624,87$ & $854.848,40$ & $519.090,50$ & $332.685,97$ \\
R19 & $2.883 .520,92$ & $1.666 .098,39$ & $733.296,70$ & $484.125,83$ \\
R20 & $1.865 .978,84$ & $1.059 .875,98$ & $464.930,00$ & $341.172,86$ \\
R22 & $576.041,65$ & 0,00 & 0,00 & $576.041,65$ \\
R23 & $476.220,04$ & 0,00 & 0,00 & $476.220,04$ \\
R24 & $813.378,10$ & 0,00 & 0,00 & $813.378,10$ \\
R27 & $1.723 .963,58$ & $864.222,94$ & $537.340,20$ & $322.400,44$ \\
R29 & $318.856,39$ & 0,00 & 0,00 & $318.856,39$ \\
R31 & $7.207 .644,81$ & $4.003 .125,93$ & $2.576 .996,10$ & $443.344,30$ \\
R33 & $1.135 .585,69$ & $267.771,11$ & $342.585,60$ & $525.228,99$ \\
R34 & $1.106 .594,38$ & 0,00 & 0,00 & $1.106 .594,38$ \\
R36 & $2.306 .816,74$ & $1.270 .594,66$ & $598.437,90$ & $437.784,18$ \\
R37 & $4.393 .577,72$ & $2.258 .738,31$ & $1.364 .897,20$ & $769.942,22$ \\
R38 & $8.159 .501,49$ & $4.489 .357,72$ & $2.910 .073,50$ & $760.070,27$ \\
R39 & $1.723 .963,58$ & $864.222,94$ & $548.812,10$ & $310.928,54$ \\
R42 & $851.689,27$ & 0,00 & 0,00 & $851.689,27$ \\
R43 & $892.224,05$ & 0,00 & 0,00 & $892.224,05$ \\
& & & &
\end{tabular}

Nota: 1. Variable en colones corrientes. 
Fuente: elaboración propia.

CUADRO 12

AJUSTES DE LA VARIABLE POR RUTA

\begin{tabular}{|c|c|c|c|}
\hline Ruta & $\begin{array}{c}\text { Valor de la } \\
\text { variable }\end{array}$ & $\begin{array}{l}\text { Cambio por } \\
\text { holgura }\end{array}$ & Valor deseado \\
\hline $\mathrm{R} 1$ & 0,19 & 0,08 & 0,27 \\
\hline $\mathrm{R} 2$ & 0,29 & 0,00 & 0,29 \\
\hline R3 & 0,09 & 0,00 & 0,09 \\
\hline $\mathrm{R} 4$ & 0,20 & 0,00 & 0,20 \\
\hline $\mathrm{R} 6$ & 0,20 & 0,12 & 0,32 \\
\hline R7 & 0,01 & 0,13 & 0,14 \\
\hline R9 & 0,45 & 0,00 & 0,45 \\
\hline $\mathrm{R} 10$ & 0,11 & 0,10 & 0,21 \\
\hline $\mathrm{R} 12$ & 0,33 & 0,00 & 0,33 \\
\hline $\mathrm{R} 14$ & 0,13 & 0,09 & 0,22 \\
\hline $\mathrm{R} 15$ & 0,29 & 0,00 & 0,29 \\
\hline $\mathrm{R} 17$ & 0,05 & 0,14 & 0,19 \\
\hline $\mathrm{R} 18$ & 0,07 & 0,13 & 0,20 \\
\hline R19 & 0,15 & 0,07 & 0,22 \\
\hline $\mathrm{R} 20$ & 0,17 & 0,03 & 0,20 \\
\hline $\mathrm{R} 22$ & 0,03 & 0,00 & 0,03 \\
\hline R23 & 0,22 & 0,00 & 0,22 \\
\hline $\mathrm{R} 24$ & 0,22 & 0,00 & 0,22 \\
\hline $\mathrm{R} 27$ & 0,13 & 0,09 & 0,22 \\
\hline R29 & 0,22 & 0,00 & 0,22 \\
\hline R31 & 0,22 & 0,03 & 0,25 \\
\hline R33 & 0,22 & 0,00 & 0,22 \\
\hline R34 & 0,40 & 0,00 & 0,40 \\
\hline R36 & 0,10 & 0,11 & 0,21 \\
\hline R37 & 0,24 & 0,03 & 0,27 \\
\hline R38 & 0,28 & 0,00 & 0,28 \\
\hline R39 & 0,12 & 0,09 & 0,21 \\
\hline $\mathrm{R} 42$ & 0,29 & 0,00 & 0,29 \\
\hline R43 & 0,07 & 0,00 & 0,07 \\
\hline
\end{tabular}


Fuente: elaboración propia.

CUADRO 13

AJUSTES DE LA VARIABLE POR RUTA

\begin{tabular}{cccc}
\hline Ruta & $\begin{array}{c}\text { Valor de la } \\
\text { variable }\end{array}$ & $\begin{array}{c}\text { Cambio por } \\
\text { holgura }\end{array}$ & Valor deseado \\
\hline R1 & $3.983,28$ & 0,00 & $3.983,28$ \\
R2 & $5.201,90$ & 0,00 & $5.201,90$ \\
R3 & $1.797,76$ & $1.987,34$ & $3.785,10$ \\
R4 & $3.339,75$ & 0,00 & $3.339,75$ \\
R6 & 804,87 & 0,00 & 804,87 \\
R7 & $1.004,64$ & $2.547,07$ & $3.551,71$ \\
R9 & $3.317,90$ & 0,00 & $3.317,90$ \\
R10 & $2.065,05$ & $1.720,05$ & $3.785,10$ \\
R12 & $4.414,14$ & 0,00 & $4.414,14$ \\
R14 & $2.910,79$ & 987,92 & $3.898,71$ \\
R15 & $6.020,92$ & 0,00 & $6.020,92$ \\
R17 & 899,25 & $2.485,24$ & $3.384,49$ \\
R18 & $1.210,28$ & 474,66 & $1.684,94$ \\
R19 & $2.840,67$ & $1.188,51$ & $4.029,18$ \\
R20 & $3.456,56$ & 104,57 & $3.561,13$ \\
R22 & 568,03 & 0,00 & 568,03 \\
R23 & $4.082,17$ & 0,00 & $4.082,17$ \\
R24 & $1.534,67$ & 0,00 & $1.534,67$ \\
R27 & $1.400,92$ & 0,00 & $1.400,92$ \\
R29 & $1.839,54$ & 0,00 & $1.839,54$ \\
R31 & $4.516,05$ & 0,00 & $4.516,05$ \\
R33 & $3.870,72$ & 0,00 & $3.870,72$ \\
R34 & $2.545,33$ & 0,00 & $2.545,33$ \\
R36 & $1.917,62$ & $2.016,09$ & $3.933,71$ \\
R37 & $4.926,63$ & 0,00 & $4.926,63$ \\
R38 & $4.456,64$ & 0,00 & $4.456,64$ \\
R39 & $2.176,42$ & 0,00 & $2.176,42$ \\
R42 & $5.160,95$ & 0,00 & $5.160,95$ \\
R43 & $1.331,05$ & 0,00 & $1.331,05$ \\
& & & \\
\hline & & &
\end{tabular}


Anexo 3

Pares de comparación para las rutas ineficientes

CUADRO 14

Pares de comparación para las rutas ineficientes

\begin{tabular}{llllllllll}
\hline Ruta/ & $\lambda_{\mathrm{R} 4}$ & $\lambda_{\mathrm{R} 6}$ & $\lambda_{\mathrm{R} 12}$ & $\lambda_{\mathrm{R} 15}$ & $\lambda_{\mathrm{R} 23}$ & $\lambda_{\mathrm{R} 29}$ & $\lambda_{\mathrm{R} 34}$ & $\lambda_{\mathrm{R} 42}$ & $\lambda_{\mathrm{R} 43}$ \\
\hline Pares ${ }^{1}$ & 0,3649 & 0,0000 & 0,5182 & 0,0000 & 0,1168 & 0,0000 & 0,0000 & 0,0000 & 0,0000 \\
R1 & 0,0000 & 0,0000 & 0,0000 & 0,0476 & 0,0000 & 0,0000 & 0,0000 & 0,9524 & 0,0000 \\
R3 & 0,4001 & 0,0000 & 0,0000 & 0,0000 & 0,5999 & 0,0000 & 0,0000 & 0,0000 & 0,0000 \\
R7 & 0,7144 & 0,0000 & 0,0000 & 0,0000 & 0,2855 & 0,0000 & 0,0000 & 0,0000 & 0,0000 \\
R10 & 0,4001 & 0,0000 & 0,0000 & 0,0000 & 0,5999 & 0,0000 & 0,0000 & 0,0000 & 0,0000 \\
R14 & 0,2471 & 0,0000 & 0,0000 & 0,0000 & 0,7529 & 0,0000 & 0,0000 & 0,0000 & 0,0000 \\
R17 & 0,9397 & 0,0000 & 0,0000 & 0,0000 & 0,0603 & 0,0000 & 0,0000 & 0,0000 & 0,0000 \\
R18 & 0,0000 & 0,1494 & 0,0000 & 0,0000 & 0,0000 & 0,8505 & 0,0000 & 0,0000 & 0,0000 \\
R19 & 0,0000 & 0,0000 & 0,0000 & 0,0000 & 0,9807 & 0,0000 & 0,0000 & 0,0000 & 0,0193 \\
R20 & 0,7018 & 0,0000 & 0,0000 & 0,0000 & 0,2982 & 0,0000 & 0,0000 & 0,0000 & 0,0000 \\
R27 & 0,0000 & 0,0379 & 0,0000 & 0,0000 & 0,0000 & 0,9621 & 0,0000 & 0,0000 & 0,0000 \\
R31 & 0,0000 & 0,0000 & 0,0000 & 0,0000 & 0,5978 & 0,0000 & 0,0000 & 0,4021 & 0,0000 \\
R33 & 0,0000 & 0,0000 & 0,0000 & 0,0000 & 0,9043 & 0,0000 & 0,0427 & 0,0000 & 0,0530 \\
R36 & 0,2000 & 0,0000 & 0,0000 & 0,0000 & 0,8000 & 0,0000 & 0,0000 & 0,0000 & 0,0000 \\
R37 & 0,0000 & 0,0000 & 0,0000 & 0,0000 & 0,2172 & 0,0000 & 0,0000 & 0,7828 & 0,0000 \\
R38 & 0,0000 & 0,0000 & 0,0000 & 0,0000 & 0,3334 & 0,0000 & 0,1318 & 0,5348 & 0,0000 \\
R39 & 0,2245 & 0,0000 & 0,0000 & 0,0000 & 0,0000 & 0,7755 & 0,0000 & 0,0000 & 0,0000 \\
\hline
\end{tabular}

Nota: 1. Los referentes corresponden a las rutas eficientes.

Fuente: elaboración propia.

Todos los derechos reservados. Universidad de Costa Rica. Este artículo se encuentra licenciado con Creative Commons Reconocimiento-NoComercial-SinObraDerivada 3.0 Costa Rica. Para mayor información escribir a revista.iice@ucr.ac.cr 